\title{
Are Adult Physiques Geometrically Similar? The Dangers of Allometric Scaling Using Body Mass Power Laws
}

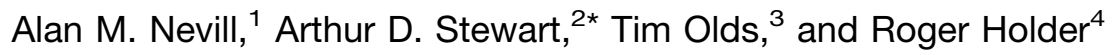 \\ ${ }^{1}$ School of Sport, Performing Arts and Leisure, University of Wolverhampton, Walsall, WS1 3BD UK \\ ${ }^{2}$ Department of Biomedical Sciences, University of Aberdeen, Aberdeen, Scotland, AB25 22D UK \\ ${ }^{3}$ School of Physical Education, Exercise and Sports Studies, University of South Australia, Underdale, South \\ Australia, 5032 Australia \\ ${ }^{4}$ School of Mathematics and Statistics, University of Birmingham, Birmingham, B15 2TT UK
}

KEY WORDS $\quad$ corrected girth; anthropometry; allometric scaling; athlete

\begin{abstract}
Human physique classification by somatotype assumes that adult humans are geometric similar to each other. However, this assumption has yet to be adequately tested in athletic and nonexercising human populations. In this study, we assessed this assumption by comparing the mass exponents associated with girth measurements taken at 13 different sites throughout the body in 478 subjects (279 athletic subjects, and 199 nonexercising controls). Corrected girths which account for subcutaneous adipose tissue at the upper arm, thigh, and calf sites, and which simulate muscle circumference, were also calculated. If subjects are geometrically similar to each other, girth exponents should be approximately proportional to $\mathrm{M}^{1 / 3}$, where $\mathrm{M}$ is the subjects' body mass. This study confirms that human adult physiques are not geometrically similar to each other. In both athletic subjects
\end{abstract}

In 1921, the first systematic anthropometric assessment of humans was proposed as a rationale for testing human efficiency by quantifying body tissues in a sample of barbers, butchers, blacksmiths, and gymnastics instructors (Matiegka, 1921). The author recognized that "above average stature is not always an indication of physical superiority." In the decades which followed, other physique descriptions referred to as "somatotyping" were proposed (Sheldon et al., 1940; Parnell, 1954), and these were later revised by Heath and Carter (1967). The latter comprised the primary physique assessment tool still in use today, based on 10 anthropometric measurements. These generate the coordinates for a tripolar schema involving fatness, musculoskeletal robustness, and mass in relation to stature. The authors stated that the resulting somatotype "is a measure of shape, not size," with the inherent assumption that adults of differing size are geometrically similar. Subsequent decades were witness to a rapid increase in the prevalence and severity of obesity, and to unprecedented muscle gains due to more sophisticated training or pharmacological interven- and nonexercising controls, body circumferences/limb girths develop at a greater rate than that anticipated by geometric similarity in fleshy sites containing both muscle and fat (upper arms and legs), and less than anticipated in bony sites (head, wrists, and ankles). Interestingly, head girths appear to remain almost constant, irrespective of subjects' body size/mass. The results also suggest that thigh muscle girths of athletes and controls increase at a greater rate than that predicted by geometric similarity, proportional to body mass ( $\mathrm{M}^{0.439}$ and $\mathrm{M}^{0.377}$, respectively). These systematic deviations from geometric similarity have serious implications for the allometric scaling of variables such as energy expenditure, oxygen uptake, anaerobic power, and thermodynamic or anthropometric studies involving individuals of differing size. Am J Phys Anthropol 124:177-182, 2004. @ 2004 Wiley-Liss, Inc.

tion. As such, humanity exhibits an "expanding universe" of possible physiques, in which the boundaries are being continually pushed back. This has enabled an increased variance in physique which, with sufficient data, can test the hypothesis of whether geometric similarity is observed in humans.

A useful insight into the issue is provided by comparison of different animal species. Larger mammals expend more energy than smaller mammals due to their larger energy cost of metabolism and locomotion. However, if energy expenditure is expressed as a simple ratio standard, per unit body mass, smaller mammals consume more energy per unit body mass than larger mammals. A theoretical

\footnotetext{
*Correspondence to: Arthur D. Stewart, Ph.D., Department of Biomedical Sciences, University of Aberdeen, Aberdeen, AB25 22D UK. E-mail: a.d.stewart@abdn.ac.uk

Received 21 February 2003; accepted 22 April 2003.

DOI 10.1002/ajpa.10351

Published online 19 September 2003 in Wiley InterScience (www. interscience.wiley.com).
} 
explanation for this observation is based on the assumption that energy expenditure of mammals obeys the surface-area law, i.e., energy expenditure is proportional to $\mathrm{M}^{0.67}$, where $\mathrm{M}$ is body mass. However, a large number of studies, both human and animal, reported mass exponents greater than 0.67 , often closer to the parameter 0.75 proposed by Kleiber (1932, 1947). Since then, various authors have attempted to explain these inflated exponents using a variety of different theories, such as the model of elastic similarity proposed by McMahon (1973). However, Heusner (1987) still concluded that "to date there is no biologically satisfactory theoretical explanation of the 0.75 power of mass."

Recent articles by Darveau et al. (2002) and Weibel (2002) provided a valuable new insight into this conundrum. They highlighted the dangers of adopting the simple power law $\mathrm{MR}=\mathrm{a} \cdot \mathrm{M}^{\mathrm{b}}$ to explain the association between metabolic rate (MR) and body mass (M), where $a$ and $b$ are referred to as the scaling constant and scaling exponent, respectively. Both articles provided persuasive evidence to explain why this simple "allometric" or power law was inadequate to model the rather complex relationship between metabolic rate and body mass. They based their arguments on the principle that multiple control sites or processes (e.g., pulmonary diffusion, cardiac output, ATP synthesis for muscle work) all contribute to metabolic pathways. They expressed this relationship as a weighted sum of mass exponents, given by:

$$
\mathrm{MR}=\mathrm{a} \cdot \sum \mathrm{c}_{\mathrm{i}} \mathrm{M}^{\mathrm{b}_{\mathrm{i}}}
$$

where $a$ is the scaling constant, and $c_{i}$ and $b_{i}$ are the control coefficients and scaling exponents for the ith control site or process, respectively. However, all the mass exponents, $b_{i}$, adopted by Darveau et al. (2002) in their calculations/simulations, were greater than 0.67. For example, the mass exponents used to estimate maximum metabolic rate (MMR) ranged from $\mathrm{b}=0.77$ (actomyosin ATPase) to $\mathrm{b}=1.08$ (pulmonary diffusion). As far as we could ascertain, Darveau et al. (2002) made no attempt to provide a biological explanation as to why any of these mass exponents were all greater than 0.67 , either individually or collectively.

An explanation for these possibly inflated (or biased) mass exponents could be due to mammals failing to conform to a fundamental assumption associated with fitting power laws. In order to estimate valid and unbiased body mass exponents when investigating the power-law association between a physiological output variable (e.g., cardiac output) and a body size variable (e.g., body mass), all subjects used in the calculations/simulations are assumed to be geometrically similar to each other. For example, if subjects are geometrically similar, individual body components (e.g., homologous muscles, hearts, lungs) should have masses proportional to body mass (M), cross-sectional or surface areas pro- portional to $\mathrm{M}^{0.67}$, and linear dimensions, such as heights or limb girths, proportional to $\mathrm{M}^{0.33}$.

However, Alexander et al. (1981) reported that in a wide variety of different species (mammals) ranging from the shrew Sorex to the elephant Loxodonta, the proximal leg muscle mass had a greater proportion of muscle mass to body mass $\left(\mathrm{M}^{1.1}\right)$. In humans, there is similar evidence to suggest that in both adolescents (Nevill, 1994a) and athletes (Nevill et al., 2003), the thigh leg muscle mass also increases at a greater proportion to body mass, estimated as $\mathrm{M}^{1.1}$ and $\mathrm{M}^{1.38}$, respectively.

If the subjects in any study used to estimate the mass exponents exhibit a similar disproportionate increase in muscle mass, an important assumption associated with fitting power laws will have been violated, leading to biased and possibly inflated mass exponents.

Although the studies by Alexander et al. (1981), Nevill (1994a), and Nevill et al. (2003) provided evidence that animals and adolescent, athletic humans are not geometrically similar to each other, no study has been undertaken to establish whether the principle of geometric similarity is appropriate in adult (nonathletic) human populations. Hence the purpose of the present study was to assess whether adult humans are geometrically similar, by comparing the mass exponents associated with girth measurements taken at different sites throughout the body in normal (control) and athletic subjects. If we find evidence that adult humans are not geometrically similar, those sites found to increase disproportionately with body mass might provide us with a plausible mechanism to explain why numerous studies reported inflated mass exponents $(b \geq 0.75)$ when modeling maximum and basal metabolic rate.

\section{METHODS}

\section{Subjects}

All subjects $(\mathrm{n}=478)$ were adults (18 years or over), and included untrained controls and athletes in a variety of sports competing at club, regional, and national levels. They were measured by anthropometrists accredited by the International Society for the Advancement of Kinanthropometry (ISAK) at the Universities of Aberdeen (UK) and South Australia. All measurements were made subject to informed consent and in accordance with the ethical requirements of the local institutions. The physical characteristics (mean \pm SD) of subjects, by training status and sex, are given in Table 1. Healthy male and female subjects were classified according to training status (athlete vs. control), with athletes being further subdivided according to type of sport (endurance, e.g. orienteering; speed, e.g., long jumping; strength, e.g. power lifting; and other games, e.g., hockey). 
TABLE 1. Physical characteristics (mean $\pm S D$ ) of control and athletic groups by sex

\begin{tabular}{|c|c|c|c|c|c|c|c|c|c|c|c|c|c|c|}
\hline \multirow[b]{2}{*}{ Group } & \multicolumn{7}{|c|}{ Male } & \multicolumn{7}{|c|}{ Female } \\
\hline & $\mathrm{n}$ & Age & $\mathrm{SD}$ & Mass & $\mathrm{SD}$ & Height & $\mathrm{SD}$ & $\mathrm{n}$ & Age & $\mathrm{SD}$ & Mass & $\mathrm{SD}$ & Height & $\mathrm{SD}$ \\
\hline Control & 95 & 30.0 & 9.6 & 75.2 & 9.2 & 178.5 & 8.2 & 104 & 30.2 & 8.8 & 61.7 & 8.7 & 166.0 & 6.1 \\
\hline Endurance & 21 & 31.7 & 10.2 & 76.0 & 12.4 & 176.6 & 5.8 & 26 & 34.5 & 8.6 & 57.3 & 5.3 & 165.2 & 5.3 \\
\hline Games & 47 & 28.1 & 9.7 & 80.8 & 13.1 & 184.7 & 11.4 & 44 & 25.5 & 8.1 & 63.6 & 10.3 & 170.7 & 10.3 \\
\hline Speed & 96 & 24.8 & 4.9 & 91.5 & 14.6 & 184.2 & 8.0 & 2 & 23.6 & 6.2 & 58.3 & 7.7 & 167.1 & 6.2 \\
\hline Strength & $\begin{array}{r}28 \\
287\end{array}$ & 28.0 & 6.6 & 86.7 & 11.4 & 178.7 & 6.5 & $\begin{array}{r}5 \\
191\end{array}$ & 25.5 & 7.6 & 55.3 & 6.9 & 167.3 & 6.5 \\
\hline
\end{tabular}
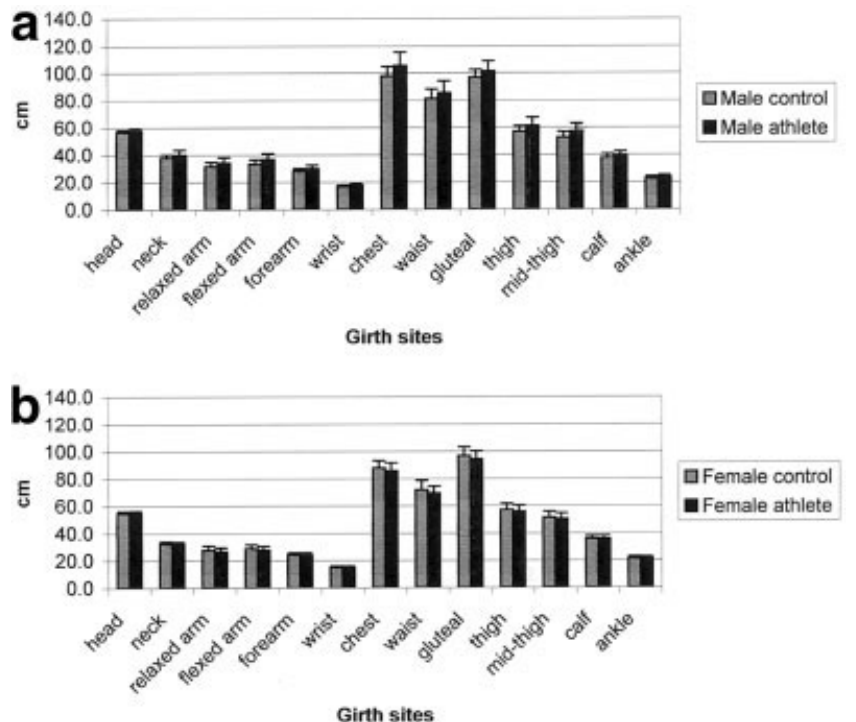

Fig. 1. a: Mean ( \pm SD) girths of male subjects for each site by group (control vs. athlete). b: Mean ( \pm SD) girths of female subjects for each site by group (control vs. athlete).

\section{Procedures}

In order to assess whether adult humans are geometrically similar to each other, we adopted body circumferences (girths) as our body-size dimension. All measurements were made using the sites and protocols recommended by ISAK (International Society for the Advancement of Kinanthropometry, 2001). Girths were recorded at 13 sites throughout the body, with measurements taken to the nearest $0.1 \mathrm{~cm}$ using a Lufkin W606PM anthropometric tape (Cooper Industries). The mean ( $\pm \mathrm{SD}$ ) girths of male and female subjects for each site by training status (controls vs. athletes) are given in Figure 1. In addition, skinfold thickness were measured using Harpenden calipers (British Indicators, Luton, UK).

Recognizing that these girths will include a contribution from subjects' body fat, we estimated the corrected girths at three sites where the skinfold and girths coincided. These were at upper-arm, midthigh, and calf, according to the method outlined in Stewart et al. (2002), as follows. Tissue boundaries were assumed to be circular and concentric, an assumption confirmed using magnetic resonance imaging (Tothill and Stewart, 2002). If the skin plus adipose tissue thickness is $\mathrm{d}$ and girth is denoted by $\mathrm{G}$, then the corrected girth $\mathrm{CG}$ is estimated by $\mathrm{CG}=$ $\mathrm{G}-2 \pi^{*} \mathrm{~d}$. (skinfold thicknesses are converted to $\mathrm{cm}$ for this calculation). Assuming that the skinfold caliper reading $S$ is twice the skin plus adipose tissue thickness $\mathrm{d}$, then $\mathrm{CG}=\mathrm{G}-\pi^{*} \mathrm{~S}$. Using this method, muscle girths were corrected at three sites (midthigh, upper arm, and calf) by subtracting $\pi$ multiplied by the skinfold thickness of the thigh, triceps, and medial calf, respectively.

\section{Statistical methods}

To establish whether the girth measurements recorded at each site increased in proportion to body mass $(\mathrm{M})$, we adopted the following allometric model similar to that proposed by Nevill and Holder (1994) when modeling maximum oxygen uptake:

$$
\mathrm{G}=\mathrm{a}_{\mathrm{i}} \cdot \mathrm{M}^{\mathrm{b}_{\mathrm{i}}} \cdot \exp \left(\mathrm{c}_{\mathrm{i}} \cdot \operatorname{age}+\mathrm{d}_{\mathrm{i}} \cdot \operatorname{age}^{2}\right),
$$

where $a_{i}$ and $b_{i}$ are the scaling constant and scaling exponents for each site ( $i=1,2,13)$, respectively. Age was incorporated into the model as a quadratic polynomial (using both age and age ${ }^{2}$ terms) to accommodate the likelihood that girths may peak sometime during adulthood and decline thereafter. The model can be linearized with a log-transformation, and multiple regression can be used to estimate the mass exponents separately for each site by "sex" and "training status" (controls vs. athletes) after controlling for age.

The same model can be used to describe corrected girths (CG), and after a log transformation, analysis of covariance (ANCOVA) can be used to compare differences between "sex" and "type of sport" (in this case, using the "sport" categories described in Table 1) by allowing $\log _{e}\left(a_{i}\right)$ and $b_{i}$ to vary for each level of "type of sport" and "sex," and using age and age ${ }^{2}$ as covariates. Again, based on the principle of geometric similarity, all three corrected muscle girths should increase in proportion to $\mathrm{M}^{0.33}$. Also based on the principle of geometric similarity, we can estimate that the muscle mass at each site will be proportional to corrected girth $(\mathrm{CG})^{3}$ or $\left(\mathrm{M}^{\mathrm{b}_{\mathrm{i}}}\right)^{3}$.

\section{RESULTS}

\section{Mass exponents of uncorrected girths}

The estimated mass exponents $\left(b_{j}\right)$ for all 13 girth sites by "sex" and "training status" (controls vs. athletes), having been adjusted for differences in age, are given in Table 2.

\section{Mass exponents of corrected thigh girths}

The ANCOVA of the log-transformed corrected thigh girths identified only one significant interac- 
TABLE 2. Estimated mass exponents $\left(b_{i}\right)$ for all 13 girth sites by "sex" and "training status" (controls vs. athletes), having been adjusted for age

\begin{tabular}{|c|c|c|c|c|c|c|c|c|}
\hline \multirow{2}{*}{$\begin{array}{l}\text { Mass exponents }\left(\mathrm{b}_{\mathrm{i}}\right) \\
\text { (adjusted for age) }\end{array}$} & \multicolumn{2}{|c|}{ Male (n = 95) } & \multicolumn{2}{|c|}{ Male $(\mathrm{n}=192)$} & \multicolumn{2}{|c|}{ Female $(\mathrm{n}=104)$} & \multicolumn{2}{|c|}{ Female $(\mathrm{n}=87)$} \\
\hline & Control & SEE & Athlete & SEE & Control & SEE & Athlete & SEE \\
\hline Head girth & 0.10 & 0.018 & 0.13 & 0.010 & 0.05 & 0.018 & 0.11 & 0.020 \\
\hline Neck girth & 0.32 & 0.029 & 0.43 & 0.020 & 0.25 & 0.027 & 0.25 & 0.027 \\
\hline Relaxed arm girth & 0.52 & 0.055 & 0.60 & 0.029 & 0.59 & 0.048 & 0.52 & 0.049 \\
\hline Flexed arm girth & 0.51 & 0.051 & 0.55 & 0.028 & 0.55 & 0.043 & 0.49 & 0.050 \\
\hline Forearm girth & 0.42 & 0.031 & 0.41 & 0.021 & 0.37 & 0.027 & 0.44 & 0.033 \\
\hline Wrist girth & 0.27 & 0.029 & 0.31 & 0.015 & 0.24 & 0.033 & 0.32 & 0.026 \\
\hline Chest girth & 0.42 & 0.034 & 0.49 & 0.020 & 0.34 & 0.029 & 0.41 & 0.031 \\
\hline Waist girth & 0.37 & 0.036 & 0.51 & 0.020 & 0.49 & 0.035 & 0.42 & 0.028 \\
\hline Gluteal girth & 0.38 & 0.020 & 0.40 & 0.013 & 0.41 & 0.025 & 0.37 & 0.027 \\
\hline Thigh girth & 0.41 & 0.031 & 0.53 & 0.018 & 0.49 & 0.031 & 0.46 & 0.038 \\
\hline Midthigh girth & 0.46 & 0.040 & 0.51 & 0.020 & 0.50 & 0.035 & 0.47 & 0.043 \\
\hline Calf girth & 0.41 & 0.043 & 0.37 & 0.020 & 0.36 & 0.033 & 0.41 & 0.035 \\
\hline Ankle girth & 0.32 & 0.044 & 0.33 & 0.021 & 0.31 & 0.034 & 0.37 & 0.030 \\
\hline Median & 0.41 & & 0.43 & & 0.37 & & 0.41 & \\
\hline
\end{tabular}

TABLE 3. Estimated mass exponents for corrected midthigh muscle girths by "type of sport," plus standard error of estimate, 95\% confidence intervals, and estimated muscle mass exponents $\left(3 * \mathrm{~b}_{\mathrm{i}}\right)$

Estimated midthigh muscle girth mass exponents (controlled for age)

\begin{tabular}{lrcccc}
\hline \multicolumn{1}{c}{ Sport type } & $\mathrm{n}$ & $\mathrm{b}_{\mathrm{i}}$ & SEE & $95 \%$ CI & $3 * \mathrm{~b}_{\mathrm{i}}$ \\
\hline Control & 199 & 0.377 & 0.031 & $0.316-0.438$ & 1.13 \\
Endurance & 47 & 0.321 & 0.054 & $0.214-0.428$ & 0.96 \\
Game & 91 & 0.470 & 0.035 & $0.401-0.540$ & 1.41 \\
Speed & 108 & 0.498 & 0.031 & $0.437-0.558$ & 1.49 \\
Strength & 33 & 0.465 & 0.057 & $0.354-0.577$ & 1.40 \\
& 478 & & & & \\
\hline
\end{tabular}

tion, that being between "types of sport" and " $\log _{e}(\mathrm{M})$," confirming that the mass exponents for corrected thigh girths varied significantly between different "types of sport" $(P<0.01)$. The estimated mass exponents $\left(b_{i}\right)$ are given in Table 3 , together with standard errors (SEE), 95\% confidence intervals (CI), and estimated muscle-mass exponents, given by $3 * b_{i}$.

The mean mass exponent for all four "types of sport" was $b_{i}=0.439$. The confidence intervals of the three groups (speed, strength, and other games) all preclude the theoretical mass exponent, $\mathrm{b}=0.33$. Only the confidence intervals of the endurance and control groups did not preclude this theoretical parameter. The ANCOVA also identified significant "sex" and "type of sport" main effects, with female thigh muscle girths being $3.8 \%$ less than male girths $(P<0.001)$ having controlled for differences in both $M$ and age. However, due to the significant "sport" by " $\log _{e}(M)$ " interaction, reporting the differences due to the main effect "type of sport" would be misleading. The age and age ${ }^{2}$ slope parameters were $\mathrm{c}=$ 0.0025 and $d=-0.000066$, respectively, from which we can estimate, using elementary differential calculus, that thigh muscle girths should peak at age $=$ $-\mathrm{c} /(2 \cdot \mathrm{d})=0.0025 / 0.000132=19$ years .

\section{Mass exponents of corrected upper-arm girths}

In contrast, the ANCOVA of the log-transformed corrected arm girth identified no interactions with " $\log _{e}(\mathrm{M})$," confirming that the mass exponents were similar for all types of sports and both sexes, estimated as $\mathrm{b}=0.451$ (SEE $=0.024 ; 95 \% \mathrm{CI}, 0.404-$ 0.498). The ANCOVA also identified significant "sex" and "type of sport" main effects, with female arm corrected girths being $17.7 \%$ less than male arm girths $(P<0.001)$, and the "strength" group having $11.0 \%$ greater arm girths compared with the "control," "endurance," and "other games" groups $(P<$ $0.001)$, having adjusted for differences in the covariates $\log _{\mathrm{e}}(\mathrm{M})$ and age and age ${ }^{2}$. Again, using elementary differential calculus, we can estimated that upper-arm corrected girths peak at age $=-\mathrm{c} /(2 \cdot \mathrm{d})=$ $0.00709 / 0.00019=37$ years.

\section{Mass exponents of corrected calf girths}

Finally, the ANCOVA of log-transformed corrected calf girths identified only one significant interaction, that between "sex" and " $\log _{e}(\mathrm{M})$," indicating that the male and female calf muscle girth mass exponents differed significantly $(P<0.01)$. The calf muscle girth exponent for male subjects was $\mathrm{b}=$ 0.350 (SEE $=0.024 ; 95 \%$ CI, 0.302-0.398), compared with $\mathrm{b}=0.218(\mathrm{SEE}=0.033 ; 95 \% \mathrm{CI}, 0.154-$ 0.283) for female subjects. As with the thigh and arm corrected girths, the ANCOVA also identified significant "sex" and "type of sport" main effects. The "type of sport" main effect identified that the strength and speed groups' corrected calf girths were $3 \%$ greater than the corrected calf girths of the control and endurance groups $(P<0.05)$, having controlled for $\mathrm{M}$ and age. Again, reporting the differences due to "sex" would be misleading due to the significant "sex" by " $\log _{e}(\mathrm{M})$ " interaction. As before, we can predict corrected calf girths to peak at age = $-c /(2 \cdot d)=0.00405 / 0.000118=34$ years.

\section{DISCUSSION}

There can be no doubt that adult humans (even within athletic or nonexercising groups) are not geo- 
metrically similar to each other. Table 2 confirms that in all groups, body circumferences/limb girths develop at a greater rate than anticipated by geometric similarity in fleshy sites containing both muscle and fat (upper arms and legs), and less than anticipated in bony sites (head, wrists and ankles). Indeed, head girths would appear to remain almost constant, irrespective of subjects' body size/mass.

These findings are at least partially supported by previous research across different mammalian species (Prothero, 1992). Although Prothero (1992) observed that whole-body length, hindlimb length, and surface area did conform to geometric similarity, the author also confirmed that forelimb length and, in particular, chest girth were greater that predicted by geometric similarity (with exponents as 0.360 (SEE, 0.009) and 0.365 (SEE, 0.005), respectively). Clearly, as in the present study, the chest girths of terrestrial mammals are increasing at a significantly greater rate than anticipated by geometric similarity, although components of muscularity and adiposity were not estimated separately.

In an attempt to compare our exponents with those reported by Alexander et al. (1981), there was a need to remove the contributions of fat from our proximal/upper-leg girth measurements. This is particularly important, even in athletic females, since the thigh is a principal site for adipose tissue accumulation. Using corrected thigh girths, again we were able to confirm that mass exponents of all groups except the endurance athletes increased in excess of that $(b=0.33)$ anticipated by geometric similarity. The estimated thigh muscle-mass exponents exceeded $b=1.4$ in 3 of the 4 sporting groups (strength, speed, and games), not dissimilar to what was reported $(b=1.38)$ by Nevill et al. (2003). Given the need of endurance athletes to carry as little body mass as possible, it was not surprising to find the endurance athletes' thigh muscle mass exponent $(b=0.96)$ to be the only estimate that was marginally less than that suggested by geometric similarity. Indeed, even the control group's thigh musclemass exponent $(b=1.13)$ was greater than unity and similar to the proximal leg muscle mass exponent $(b=1.1)$ reported by Alexander et al. (1981) when studying a variety of different mammal species.

Further evidence to support the above observation that athletic and control subjects have a muscle mass greater than that predicted by geometric similarity, we calculated the allometric exponent of lowerlimb length (stature - sitting height) as a function of stature. The estimated exponent was 1.22 (SEE, 0.051 ), confirming that there exists a greater proportion of lower-limb or leg length to stature in taller individuals. Thus, with greater than geometric similarity exponents both for corrected thigh girth and lower-limb length, the estimated muscle volume and mass must also increase commensurately.

A longer limb that is also heavier requires a greater force for acceleration according to Newton's second law (force equals mass multiplied by acceleration). A longer limb (but with an equivalent mass distribution), by virtue of its segmental inertia, would have a slower frequency of oscillation, and would require greater muscle contraction to accelerate or decelerate. This, coupled with a heavier body, implies a disproportionate force production requirement, in order to facilitate agility and rapid changes in direction. Since the principal muscles providing this power are the quadriceps and hamstrings, the above process provides a plausible biomechanical explanation for the observed thigh muscle mass enlargement.

Further evidence that human muscle mass increases at a greater proportion to body mass was obtained from analyses of corrected upper-arm and calf girths. The upper-arm muscle girth exponents were found to be common for all groups (males and females; athletes and controls), estimated as $\mathrm{b}=$ 0.451 (SEE $=0.024 ; 95 \%$ CI, 0.404-0.498). As can be seen from the CI, this exponent also precludes the parameter $b=0.33$ anticipated by geometric similarity. The estimated upper-arm muscle-mass exponent, given by $3 * b_{i}$, became $b=1.35$. As for calf girths, the male corrected calf-girth exponent was $\mathrm{b}=0.35$, marginally greater that that anticipated by geometric similarity, while for females, the corrected calf-girth exponent was $b=0.218$, the only muscle site and group (females) that failed to provide evidence that the proportion of muscle mass to body mass exceeded that predicted by geometric similarity.

Clearly, the present results confirm that in athletes and nonexercising controls, as total mass increases, the proportion of muscle mass to body mass exceeds that predicted by geometric similarity. In particular, the thigh muscle mass $\left(\mathrm{M}_{\mathrm{T}}\right)$ of controls increased in proportion to body mass, $\mathrm{M}^{1.13}$. Assuming that the thigh muscles make a major and/or representative contribution to maximum oxygen uptake $\left(\mathrm{VO}_{2} \max \right)$ performance and that the contribution of thigh-muscle mass to $\mathrm{VO}_{2}$ max obeys the surface area law $\left(\mathrm{M}_{\mathrm{T}}\right)^{0.67}$, then the controls will expend a similar disproportionate increase in energy expenditure $\left(\mathrm{V}_{2} \mathrm{max}\right)$, given by

$$
\dot{\mathrm{V}} \mathrm{O}_{2} \max \sim\left(\mathrm{M}_{\mathrm{T}}\right)^{0.67} \sim\left(\mathrm{M}^{1.13}\right)^{0.67} \sim \mathrm{M}^{0.753},
$$

not dissimilar to the mass exponent $b=0.75$, proposed by Kleiber $(1932,1947)$ and Nevill (1994b).

We recognize that muscle is not the only control site or process that contributes to metabolic rate, and that other contributions must be taken into consideration when modeling MMR and basal metabolic rate (Darveau et al., 2002; Weibel, 2002), and when investigating differences in thermoregulation by sex (Wright et al., 2002) or somatotype (Hayward et al., 1986). However, by observing the lack of geometric similarity in humans, the present study highlights the dangers of fitting power laws, either collectively or separately, to multiple sites or processes 
when modeling performance variables such as muscle strength, aerobic power, cardiac output, and maximum and basal metabolic rate.

\section{CONCLUSIONS}

The results of the present study were able to confirm that the proportion of human (controls and athletes) muscle mass to body mass was greater than that predicted by geometric similarity, a finding that provides a plausible biological mechanism to explain the inflated exponents of $b \geq 0.75$, reported in numerous studies when modeling metabolic rate. The present findings highlight the dangers of using body mass power laws to scale/model performance variables recorded on individuals of different body size. For such a sample, rather than using total mass, corrected girth methodology should be explored as an alternative method of normalizing data.

\section{ACKNOWLEDGMENTS}

The authors thank Barry Ridge, Lindsay Carter, Grant Tomkinson, Shelley Kay, and Alex Mavroeidi for permission to use their data in these analyses.

\section{LITERATURE CITED}

Alexander RMN, Jayes AS, Maloiy GMO, Wathuta EM. 1981. Allometry of the leg muscles of mammals. J Zool Lond 194:539552.

Darveau CA, Suarez RK, Andrews RD, Hochachka PW. 2002. Allometric cascade as a unifying principle of body mass effects on metabolism. Nature 417:166-170.

Hayward JS, Eckerson JD, Dawson BT. 1986. Effect of mesomorphy on hyperthermia during exercise in a warm humid environment. Am J Phys Anthropol 70:11-17.

Heath BH, Carter JEL. 1967. A modified somatotype method. Am J Phys Anthropol 27:57-74.
Heusner AA. 1987. What does the power function reveal about structure and function in animals of different size? Annu Rev Physiol 49:121-133.

International Society for the Advancement of Kinanthropometry. 2001. International standards for anthropometric assessment. Underdale, South Australia: ISAK.

Kleiber M. 1932. Body size and metabolism. Hilgarida 6:315-353.

Kleiber M. 1947. Body size and metabolic rate. Physiol Rev 27: 511-541.

Mateigka J. 1921. The testing of physical efficiency. Am J Phys Anthropol 4:223-230.

McMahon T. 1973. Size and shape in biology. Elastic criteria impose limits on biological proportions, and consequently on metabolic rates. Science 174:1201-1204.

Nevill AM. 1994a. Evidence of an increasing proportion of leg muscle mass to body mass in male adolescents and its implication on performance. J Sports Sci 12:163-164.

Nevill AM. 1994b. The need to scale for differences in body size and mass: an explanation of Kleiber's 0.75 exponent. J Appl Physiol 77:2870-2873.

Nevill AM, Holder RL. 1994. Modeling maximum oxygen uptake-a case study in non-linear regression model formulation and comparison. J R Stat Soc Ser C 43:653-666.

Nevill AM, Brown D, Godfrey R, Johnson PL, Romer L, Stewart $\mathrm{AD}$, Winter EM. 2003. Modeling maximum oxygen uptake of elite endurance athletes. Med Sci Sports Exerc 35:488-494.

Parnell RW. 1954. Somatotyping by physical anthropometry. Am J Phys Anthropol 12:209-239.

Prothero J. 1992. Scaling of bodily proportions in adult terrestrial mammals. Am J Physiol 262:492-503.

Sheldon WH, Stevens SS, Tucker WS. 1940. The varieties of human physique. New York: Harper and Brothers.

Stewart AD, Stewart A, Reid D. 2002. Correcting calf circumference discriminates the incidence of falling but not bone quality by broadband ultrasound attenuation in elderly female subjects. Bone 31:195-198.

Tothill P, Stewart AD. 2002. Thigh muscle and adipose tissue volume estimation using magnetic resonance imaging and anthropometry. J Sports Sci 20:1-14.

Weibel ER. 2002. The pitfalls of power laws. Nature 417:131-132.

Wright A, Marino FE, Kay D, Micalos P, Fanning C, Cannon J, Noakes T. 2002. Influence of lean body mass on performance differences of male and female distance runners in warm, humid environments. Am J Phys Anthropol 118:285-291. 